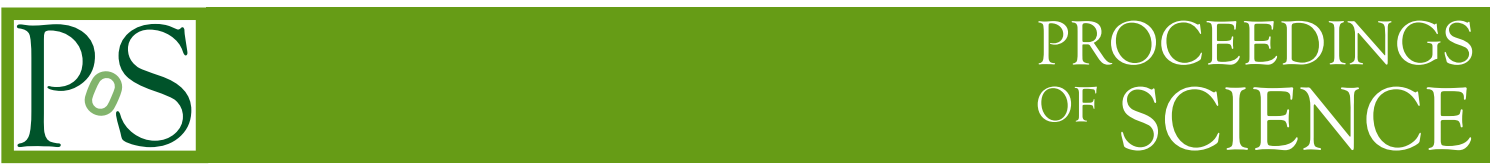

\title{
Precision Studies of Light Mesons at COMPASS
}

\author{
Bernhard Ketzer*for the COMPASS Collaboration \\ Physik Department, Technische Universität München, D-85748 Garching, Germany \\ E-mail: Bernhard.Ketzer@cern.ch
}

The COMPASS experiment at CERN's SPS investigates the structure and excitations of strongly interacting systems. Using reactions of $190 \mathrm{GeV} / c$ pions with protons and nuclear targets, mediated by the strong and electromagnetic interaction, an unprecedented statistical precision has been reached allowing new insight into the properties of light mesons. For the first time the diffractively produced $3 \pi$ final state has been analyzed simultaneously in bins of invariant mass and four-momentum transfer using a large set of 88 waves up to a total angular momentum of 6. In addition to a precise determination of the properties of known resonances and including a model-indepedent analysis of the $\pi \pi S$-wave isobar, a new narrow axial-vector state coupling strongly to $f_{0}(980)$ has been found in previously unchartered territory. By selecting reactions with very small four-momentum transfer COMPASS is able to study processes involving the exchange of quasi-real photons. These provide clean access to low-energy quantities such as radiative couplings and polarizabilities of mesons, and thus constitute a test of model predictions such as chiral perturbation theory.

XV International Conference on Hadron Spectroscopy-Hadron 2013

4-8 November 2013

Nara, Japan

\footnotetext{
*Speaker.

${ }^{\dagger}$ Present address: Helmholtz-Institut für Strahlen- und Kernphysik, Universität Bonn, D-53115 Bonn, Germany.
} 


\section{Introduction}

Despite more than fifty years of research, the light-meson excitation spectrum is still far from being understood. In the original quark model proposed in 1964 light mesons are composed of quark-antiquark $(q \bar{q})$ pairs with $L S$-coupling of total spin $S$ and orbital angular momentum $L$ between the quarks. This static model yields a surprisingly accurate description and classification of many of the light mesons known today [1]. In this model, the pseudoscalar and vector mesons are the $L=0$ combinations with total spin $S=0$ and $S=1$, respectively, yielding a total angular momentum $J=0$ or 1 . Parity and charge-conjugation parity ${ }^{1}$ are given by $P=(-1)^{L+1}$ and $C=(-1)^{L+S}$, respectively. The classification of light mesons with scalar quantum numbers $J^{P C}=0^{++}$is more difficult. One possible interpretation is that the scalars with masses below $1 \mathrm{GeV} / c^{2}$ form a nonet with an inverted mass hierarchy. The high masses of the $a_{0}(980)$ and the $f_{0}(980)$ and their large coupling to $K \bar{K}$ could be explained by interpreting them as tightly bound tetraquark states [2] or $K \bar{K}$ molecule-like objects [3]. Other interpretations favor an ordinary $q \bar{q}$ nonet consisting of $f_{0}(980), a_{0}(980), K_{0}^{*}(1430)$, and $f_{0}(1500)[4,5]$.

Quantum Chromodynamics (QCD) provided a justification for the $q \bar{q}$ rule as a possible singlet representation ${ }^{2}$ of the underlying fundamental color $S U(3)$ symmetry. Being composed of a strongly coupled system of highly relativistic light quarks $u, d$, $s$, however, calculations of the properties of hadrons using perturbative QCD are bound to fail. Furthermore, QCD allows the existence of whole new classes of mesons, including systems being composed of 4 quarks ( $q \bar{q}$ molecules or tetraquark states) and those where gluonic degrees of freedom contribute to the quantum numbers (hybrids, glueballs). Despite a long history of experiments only some isolated candidates for such states were identified (see [6-8] for recent reviews). However, the phenomenological picture is still very much dominated by the nonets of the $q \bar{q}$ model. Recently, first ab-initio calculations of hadron properties have been performed by numerically solving QCD on a Euclidean space-time lattice [911]. Similarly to the experimental situation, the quark-model states seem to be confirmed by the lattice simulations; some recent calculations also predict a full set of multiplets of non- $q \bar{q}$ states, e.g. hybrids [12].

\section{COMPASS Detector}

The COMPASS experiment at the CERN SPS set out to solve some of the open questions in light-meson spectroscopy by increasing the world data sample for exclusive reactions of highenergy $(190 \mathrm{GeV} / c)$ hadrons with various targets by a factor of ten to hundred. In COMPASS kinematics, three different mechanisms contribute to the production of a system $X$ : diffractive dissociation and central production, which can be described to proceed via the exchange of one or two Reggeons $\mathbb{R}$, respectively, between the beam hadron and the target particle $N$, and photoproduction in the Coulomb field of a nucleus (Primakoff reactions) at very low values of momentum transfer. In order to detect the final-state particles from these processes over a wide angular range with excellent resolution, COMPASS is built as a two-stage magnetic spectrometer equipped with tracking, particle identification and calorimetry, providing a very uniform acceptance for neutral

\footnotetext{
${ }^{1}$ for states composed of a quark and its own antiquark

${ }^{2}$ together with the $q q q$ system for baryons
} 

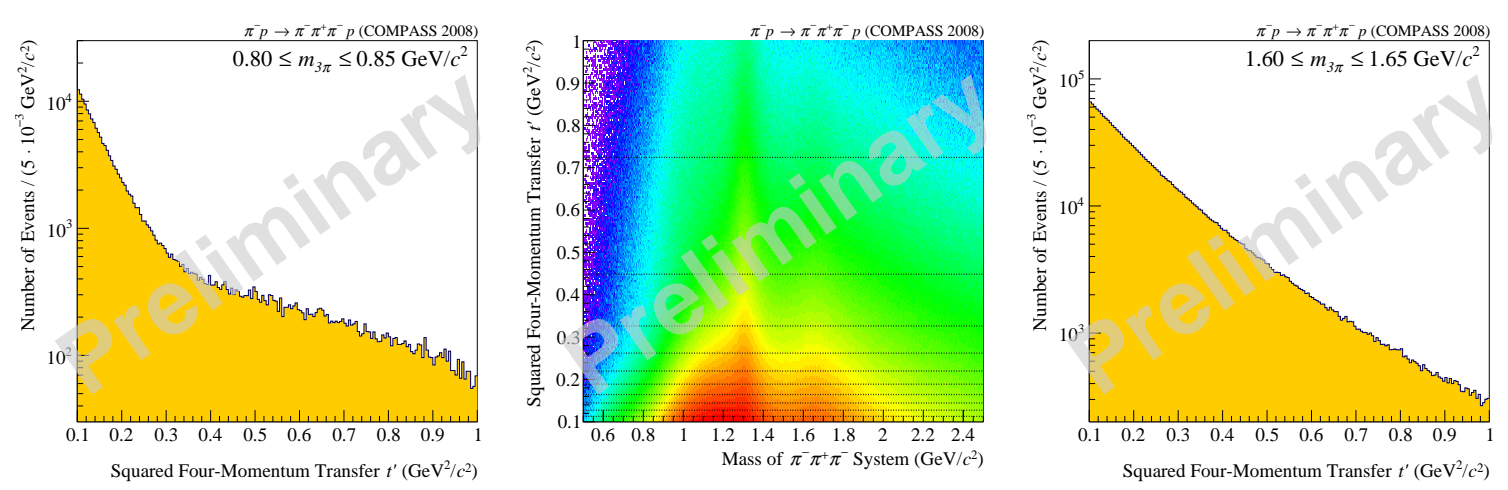

Figure 1: $3 \pi$ final state, (center) distribution of events as a function of $3 \pi$ invariant mass $m_{3 \pi}$ and 4-momentum transfer $t^{\prime}$, (left) $t^{\prime}$-distribution for $0.80 \leq m_{3 \pi} \leq 0.85 \mathrm{GeV} / c^{2}$, (right) $t^{\prime}$-distribution for $1.60 \leq m_{3 \pi} \leq 1.65 \mathrm{GeV} / c^{2}$.

and charged particles over a broad kinematical range [13]. The incoming beam particles $(\pi, p, K)$ are identified by a pair of CEDAR detectors. For the results reported in this paper a negative beam consisting of $96.8 \% \pi^{-}$was used. For the diffractive measurements a $40 \mathrm{~cm}$ long liquid-hydrogen target was employed, while for the Primakoff reactions a solid target disk made of either lead or nickel was installed. The target is surrounded by a Recoil Proton Detector (RPD) consisting of two concentric layers of scintillator bars. Events containing particles emerging at angles larger than the acceptance of the spectrometer are vetoed by a dedicated sandwich counter. The information of several trigger detectors is used in order to select exclusive events according to the physics case.

\section{Diffractive Dissociation Reactions}

\subsection{Kinematics}

For the study of diffractive dissociation reactions the trigger requires an incoming beam particle hitting the target and a signal from a recoil proton in the RPD, resulting in a 4-momentum transfer $t<-0.07 \mathrm{GeV}^{2} / c^{2}$. Exclusive events are selected by energy conservation between incoming and outgoing particles and by transverse momentum balance between incoming and outgoing particles including the recoil proton. Here we focus on the final state containing three charged pions, $\pi^{-} \pi^{-} \pi^{+}$, with about $5 \cdot 10^{7}$ events after the above-mentioned cuts, covering invariant masses up to $3 \mathrm{GeV} / c^{2}$. The corresponding analysis for the $\pi^{-} \pi^{0} \pi^{0}$ final state is covered elsewhere in these proceedings. Figure 1 (center) displays the distribution of events as a function of the invariant mass $m_{3 \pi}$ of the three-pion system and $t^{\prime}$, defined as the reduced squared 4-momentum transfer $|t|-|t|_{\min }$ to the recoiling target nucleon beyond the kinematic minimum $|t|_{\min }$. Figures 1 (left) and (right) show the projection onto the $t^{\prime}$ axis for two different bins of $m_{3 \pi}$, respectively. Evidently there is a strong correlation between these two variables, illustrating the need to analyze the data not only in bins of $m_{3 \pi}$ but also in bins of $t^{\prime}$.

\subsection{Partial-Wave Analysis}

To disentangle the resonances contributing to a given final state a partial-wave analysis (PWA) is performed using the phenomenological approach of the isobar model. In this model the produc- 
tion and the decay of a state $X$ with quantum numbers $J^{P C} M^{\varepsilon}$ factorize. Here, $M$ is the modulus of the spin projection onto a quantization axis, and $\varepsilon$ the reflectivity [14]. The decay is described as a series of sequential two-body decays into intermediate resonances (isobars), which eventually decay into the final state observed in the experiment. A partial wave is fully characterized by the quantum numbers of $X$, the isobars, their total spin and the orbital angular momentum between them. In the case of the three-pion final state, we allow for all di-pion isobars established by the PDG [1] up to a mass of $1.7 \mathrm{GeV} / c^{2}:(\pi \pi)_{S}$ wave (containing the broad $f_{0}(500)$ and the $f_{0}(1370)$ ) [15], $f_{0}(980), f_{0}(1500), f_{2}(1270), \rho(770), \rho_{3}(1690)$. The analysis makes use of an event-based extended $\log$-likelihood fit in $20 \mathrm{MeV} / c^{2}$ wide bins of $m_{3 \pi}$ and in 11 bins of $t^{\prime}$, chosen to equalize the number of events in each bin and indicated by horizontal lines in Fig. 1 (center). The fit includes the largest wave set ever used in such an analysis: 80 waves with positive reflectivity and spin up to 6 , seven waves with negative reflectivity, and a flat background wave representing three-pion phase space. Full coherence is assumed for waves with positive and negative reflectivity, respectively, while the set of positive- and negative-reflectivity waves and the flat wave are added incoherently. Only waves with negligible population have been omitted from the fit.

\subsection{Fits of Spin-Density Matrix}

The result of the PWA described in Section 3.2 is one independent spin-density matrix for each $m_{3 \pi}$ and $t^{\prime}$ bin, containing all waves used in this particular bin. In a second step, a model is applied in a $\chi^{2}$ fit to describe the mass and $t^{\prime}$-dependence of these matrices, where for computational reasons only a few waves, in our case six, are considered. For each wave the model includes resonant contributions, usually parametrized in terms of relativistic Breit-Wigner functions with dynamic widths and parameters independent of $t^{\prime}$, and non-resonant contributions added coherently, in our case parametrized by empirical functions $\exp \left[-p\left(t^{\prime}\right) q^{2}\right]$, with $p\left(t^{\prime}\right)$ a polynomial of the reduced 4-momentum transfer and $q$ the break-up momentum for 2-body decay. Figure 2 displays the result of such a fit to the spin-density matrices of the first step, displayed here as a function of $m_{3 \pi}$ and for the smallest $t^{\prime}$ bin.

Apart from the observation of known resonances with very small statistical uncertainty, the most important features are (i) the need for a second $a_{1}$ resonance (top row), (ii) the need for a second $a_{2}$ state interfering destructively with the $a_{2}(1320)$ (second row/column), both at masses close to $2 \mathrm{GeV} / c^{2}$, and (iii) the observation of a clear peak of the $1^{++} 0^{+} f_{0}(980) \pi P$ wave intensity at a mass of $1.42 \mathrm{GeV} / c^{2}$ combined with a phase motion close to $180^{\circ}$ with respect to all other waves (5th row/column). In our model, this previously unobserved object with axial-vector quantum numbers, coupling exclusively to $f_{0}(980) \pi$, is well described by a Breit-Wigner resonance, tentatively called $a_{1}(1420)$, with a rather small width of about $140 \mathrm{MeV} / c^{2}$. The interpretation of this structure as a genuine resonance or, due to its proximity to the $K^{*} \bar{K}$ threshold, as a dynamic effect [16] including rescattering of the $K \bar{K}$ to $f_{0}(980)$, is still to be clarified.

Some of the waves under study exhibit a strong $t^{\prime}$-dependence, as can be seen from Fig. 3 for the $1^{++}$waves. In the $1^{++} 0^{+} \rho \pi S$ wave (left), the broad structure is composed of the genuine $a_{1}(1260)$ resonance (which does not depend on $t^{\prime}$ ), and a background interfering destructively (upper plot: low $t^{\prime}$ ) or constructively (lower plot: high $t^{\prime}$ ) at higher masses, causing the resulting signal to change as a function of $t^{\prime}$. The 2D-analysis for the first time allows us to disentangle background and resonance components. A similar picture is observed for the $a_{1}(1420)$, shown in 


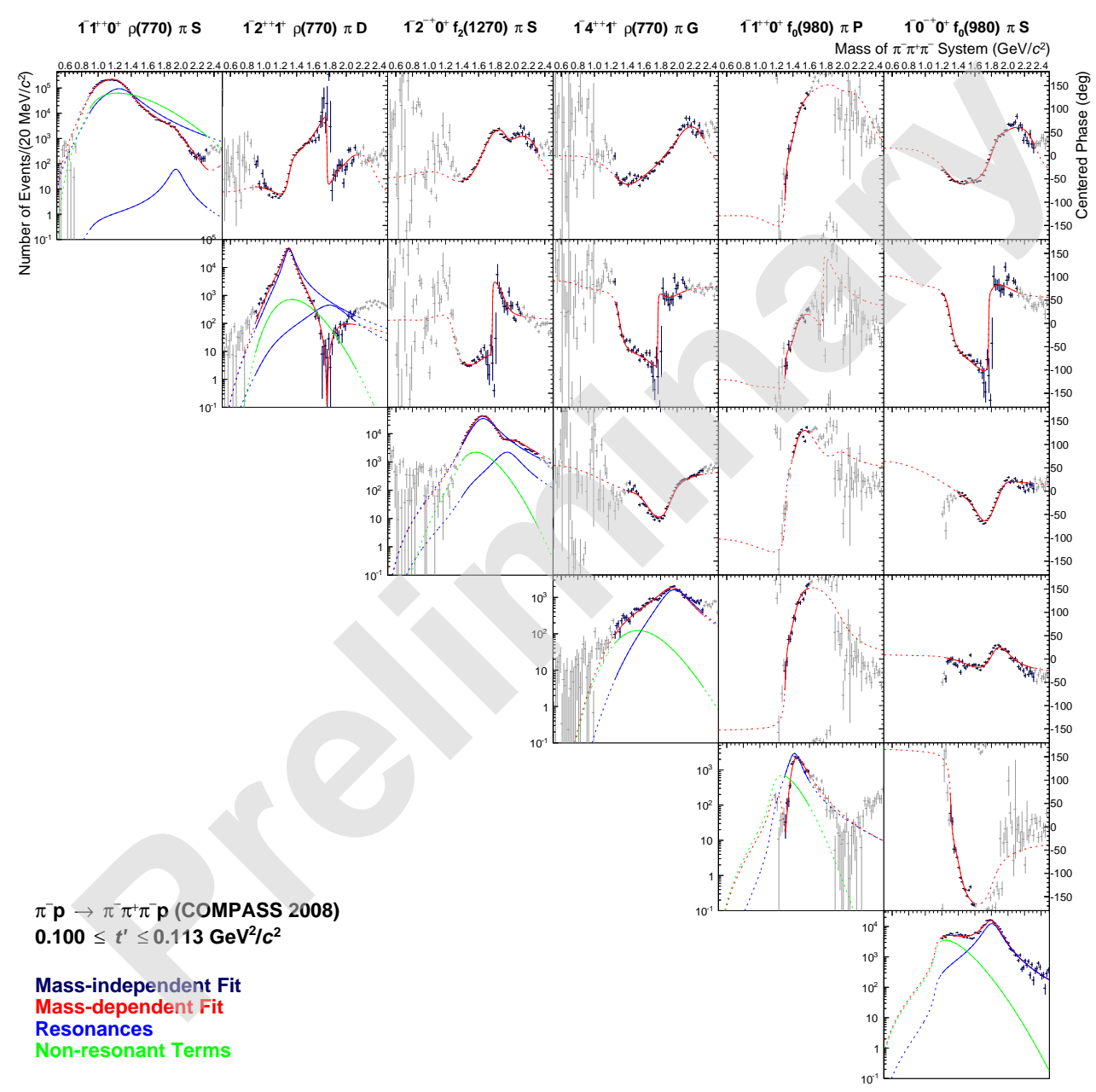

Figure 2: Mass and $t^{\prime}$-dependent fit of the spin-density matrices for six selected waves (columns labeled at the top, rows analogously), shown here for $0.100 \leq t^{\prime} \leq 0.113 \mathrm{GeV}^{2} / c^{2}$. The plots along the diagonal correspond to the intensities in the respective waves, while the off-diagonal plots show the phase differences between the wave corresponding to the column and the one corresponding to the row, respectively. The black data points are the result of the PWA in mass and $t^{\prime}$ bins, the curves indicate the fit model.

the right column of Fig. 3. Note that the fit to the spin-density matrices was performed only in the mass range indicated by solid lines.

\subsection{Model-independent Analysis of $\pi \pi S$-Wave}

In order to study the dependence of our results on the parameterization of isobars, especially the ones with $J_{\text {iso }}^{P C}=0^{++}$, we developed a method to determine the isobar dynamics directly from the data, and thus to obtain a model-independent amplitude for these isobars. In this method the fixed 2-body amplitude for $J_{\text {iso }}^{P C}=0^{++}$, which includes the parameterization by [15] of the broad $f_{0}(500)$ and $f_{0}(1370)$ as well as a Flatté ansatz for the narrow $f_{0}(980)$ and a Breit-Wigner for the $f_{0}(1500)$, is replaced for three waves, $0^{-+} 0^{+}(\pi \pi)_{S}^{*} \pi S, 1^{++} 0^{+}(\pi \pi)_{S}^{*} \pi P$, and $2^{-+} 0^{+}(\pi \pi)_{S}^{*} \pi D$, 

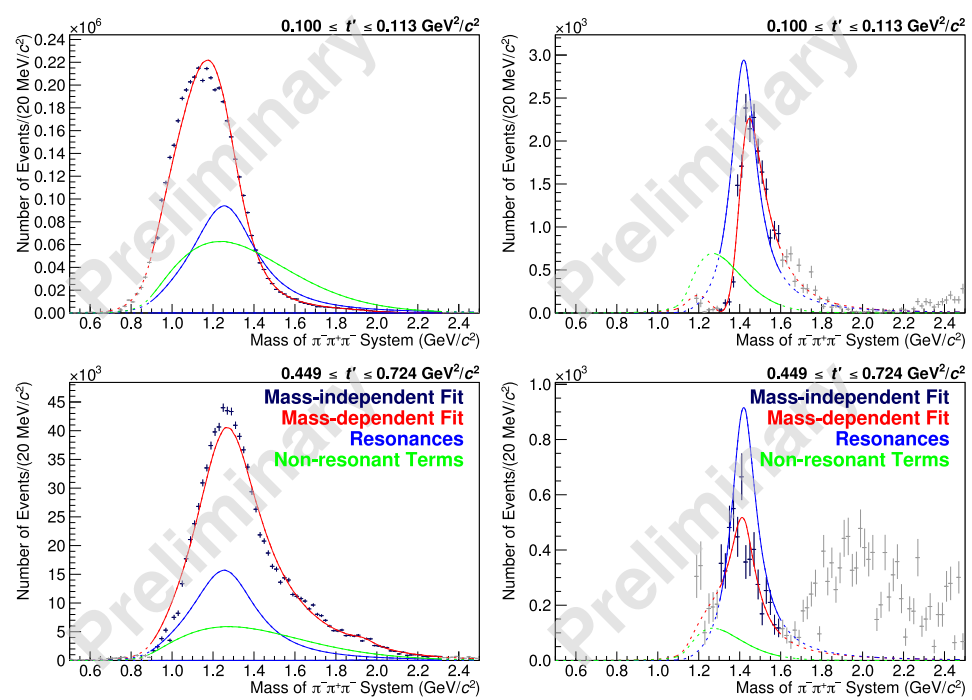

Figure 3: Intensities of (left) $1^{++} 0^{+} \rho \pi S$ and (right) $1^{++} 0^{+} f_{0}$ (980) $\pi P$ waves, for (top) the smallest and (bottom) the 2 nd highest $t^{\prime}$-bin. The curves indicate the results of the fit to the spin-density matrix: (red) total intensity, (blue) resonance contribution, (green) background.

by a set of free complex parameters in 2-body mass bins of $40 \mathrm{MeV} / c^{2}$ width $\left(10 \mathrm{MeV} / c^{2}\right.$ around the $f_{0}(980)$ ), denoted $(\pi \pi)_{S}^{*}$. There is no separation into different $0^{++}$isobars. Since the number of free parameters is much larger in this case (one complex parameter for each $m_{2 \pi}$ mass bin per wave instead of one complex parameter for each wave), the fit is performed in two $t^{\prime}$ bins only. Figure 4 depicts the result of this fit for the three waves mentioned above (columns) and for low $t^{\prime}$ (upper row) and high $t^{\prime}$ (lower row). A clear correlation of the intensity between 3-body and 2body mass is visible for all three waves. The left column shows the strong coupling of the $\pi(1800)$ to the $f_{0}(980)$ and $f_{0}(1500)$. The middle column proves the coupling of the narrow peak of the $1^{++} 0^{+}(\pi \pi)_{S}^{*} \pi P$ wave at masses around $1.4 \mathrm{GeV} / c^{2}$ to $f_{0}(980)$, which confirms its observation with the fixed isobar parameterization. In the right column the $\pi_{2}(1880)$ decaying to $f_{0}(980)$ and $f_{0}(1500)$ is clearly visible. The resonant nature of these $2 \pi$ states is also visible from the full complex amplitudes, which exhibit clear circular motions in the respective Argand diagrams. There is a striking dependence of these distributions on $t^{\prime}$ (upper vs. lower row), especially in the $1^{-+} 0^{+}(\pi \pi)_{S}^{*} \pi P$ wave (center). The very broad structure around $m_{3 \pi}=1.3 \mathrm{GeV} / c^{2}$ in the $0^{-+} 0^{+}(\pi \pi)_{S} \pi S$, commonly referred to as the $\pi(1300)$, exhibits no phase motion and a steeply falling $t^{\prime}$ dependence, which suggests it to be generated by non-resonant processes.

\section{Photoproduction Reactions}

We now turn to reactions of the incoming pion with a heavy target nucleus at very small values of 4-momentum transfer $t^{\prime} \lesssim 10^{-3} \mathrm{GeV}^{2} / c^{2}$. In this kinematic domain, the exchange of quasireal photons (Primakoff reaction) dominates over the strong interaction. The observation of these reactions gives access to the low-energy structure of hadrons, e.g. their polarizabilities, which are covered elsewhere in these proceedings, and to the coupling of hadronic resonances to photons, which is treated here. 

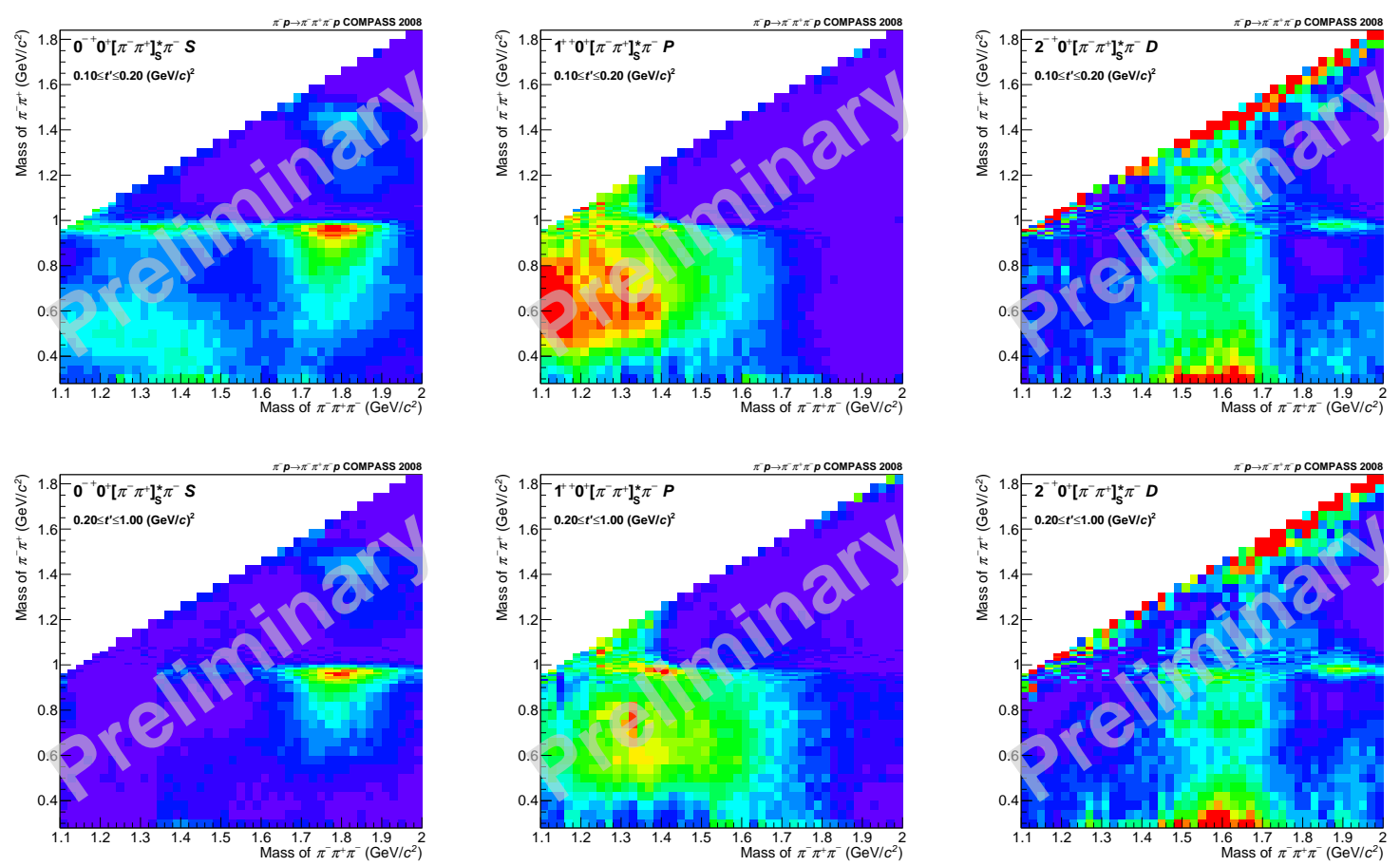

Figure 4: Intensity of waves as a function of 3-body invariant mass $m_{3 \pi}$ and 2-body mass $m_{2 \pi}$ from fit with free $0^{++}$isobar amplitude, (left) $0^{-+} 0^{+}(\pi \pi)_{S}^{*} \pi S$ wave, (center) $1^{++} 0^{+}(\pi \pi)_{S}^{*} \pi P$ wave, (right) $2^{-+} 0^{+}(\pi \pi)_{S}^{*} \pi D$ wave. (Top row) $0.1 \leq t^{\prime} \leq 0.2 \mathrm{GeV}^{2} / c^{2}$, (bottom row) $0.2 \leq t^{\prime} \leq$ $1.0 \mathrm{GeV}^{2} / c^{2}$.

We use about $10^{6}$ exclusive $\pi^{-} \pi^{-} \pi^{+}$events produced on a $\mathrm{Pb}$ target with values of $t^{\prime}<$ $0.001 \mathrm{GeV}^{2} / c^{2}$ to perform a PWA similar to the one described in Sec. 3.2. At these values of 4-momentum transfer both diffraction and photoproduction contribute to the production of a resonance $X$. With a dependence of the diffractive cross section on $t^{\prime}$ as $t^{\prime M} \exp \left(-b_{\text {diff }} t^{\prime}\right)$, however, only states with spin projection $M=0$ are produced as $t^{\prime} \rightarrow 0$, while states with $M=1$ are dominantly produced by Primakoff reactions, which depend on $t^{\prime}$ as $\exp \left(-b_{\text {prim }} t^{\prime}\right)$. The PWA then allows us to single out contributions of waves with $M=1$, providing very clean access to quasireal photoproduction and thus to the radiative coupling of resonances decaying into $3 \pi$. In the PWA clean signals in the $2^{++} 1^{+} \rho \pi D$ wave, corresponding to $\pi \gamma \rightarrow a_{2}(1320)$, and in the $2^{-+} 1^{+} f_{2} \pi S$ wave, corresponding to $\pi \gamma \rightarrow \pi_{2}(1670)$ are observed, as shown in Fig. 5.

The radiative width $\Gamma(X \rightarrow \pi \gamma)$ is extracted from the measured Primakoff cross section $\sigma_{\text {prim }}$ by relating it to the integral of the double differential cross section for Primakoff production of a wide Breit-Wigner resonance (given e.g. in [17]), calculated in the Weizsäcker-Williams equivalent-photon approximation (EPA), over the experimentally observed mass and $t^{\prime}$ range,

$$
\sigma_{\text {prim }}=\int_{m_{1}}^{m_{2}} \int_{0}^{0.001} \mathrm{~d} m \mathrm{~d} t^{\prime} \frac{\mathrm{d}^{2} \sigma}{\mathrm{d} m \mathrm{~d} t^{\prime}}=\Gamma_{0}(X \rightarrow \pi \gamma) \cdot C_{X}
$$

Here, $C_{X}$ is a normalization constant which includes the total width of the resonance under study, the partial width in the final state, and several kinematic factors related to the dynamics of the 

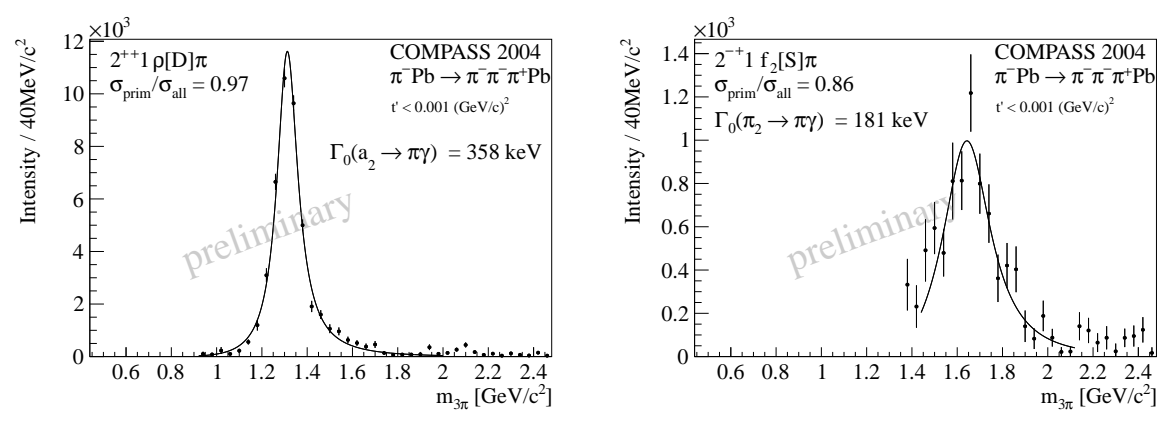

Figure 5: Intensities of (left) $2^{++} 1 \rho \pi D$ wave and (right) $2^{-+} 1 f_{2} \pi S$ wave in the Primakoff region $t^{\prime}<0.001 \mathrm{GeV}^{2} / c^{2}$, together with the Breit-Wigner fits used to determine the radiative coupling.

initial and final states, and the EPA. The curves in Fig. 5 show the result of a fit of the massdependent differential cross section in Eq. (4.1) integrated over the relevant $t^{\prime}$ range to the data points extracted from the PWA in mass bins. The integral of the fitted curve gives $\sigma_{\text {all }}$, from which the small diffractive contribution, estimated from a fit of the $t^{\prime}$-dependence of the intensities, is subtracted incoherently to give $\sigma_{\text {prim }}$. This is justified due to the fact that the phase difference between photoproduction and diffractive production is close to $90^{\circ}$ for the small values of $t^{\prime}$ used in this analysis.

The COMPASS result for the radiative width of the $a_{2}(1320)$ is $\Gamma_{0}\left(a_{2}(1320) \rightarrow \pi \gamma\right)=(358 \pm$ $6 \pm 42) \mathrm{keV}$, in fair agreement with calculations based on the vector meson dominance model [18] and a relativistic quark model [19]. For the $\pi_{2}(1670)$ the experimental result is $\Gamma_{0}\left(\pi_{2}(1670) \rightarrow\right.$ $\pi \gamma)=(181 \pm 11 \pm 27) \mathrm{keV} \cdot\left(0.56 / B R_{f_{2}} \pi\right)$, where the correction factor indicates that the branching ratio $B R_{f_{2} \pi}^{\mathrm{PDG}}=0.56$ taken from [1] was used for the quoted result, which may have to be modified when interferences between the different $3 \pi$ final states are taken into account. The systematic errors include contributions from radiative corrections, the determination of the luminosity from the decay of beam kaons, different PWA models, the subtraction of the diffractive background, and the mass-dependent parameterization of the resonance shape. The radiative width of the $\pi_{2}(1670)$ quoted here is the first experimental determination of this quantity, and differs significantly from the theoretical prediction by [20].

\section{Conclusions}

The COMPASS experiment has collected large data samples on diffractive and photon-induced reactions of light mesons. For the largest data set with $3 \pi$ final states a 2-dimensional PWA method in bins of invariant mass and 4-momentum transfer was established, which allows the disentanglement of resonant and non-resonant contributions and thus a precise determination of the parameters for a number of resonances decaying into $3 \pi$, including radial excitations of the $a_{1}(1260)$ and $a_{2}(1320)$. A new resonant structure with axial-vector quantum numbers is observed in the $1^{++} 0^{+} f_{0}(980) \pi P$ wave at a mass of $1.42 \mathrm{GeV} / c^{2}$, which we tentatively call $a_{1}(1420)$ with a BreitWigner mass of $1412-1422 \mathrm{MeV} / c^{2}$ and a rather narrow width of $130-150 \mathrm{MeV} / c^{2}$.

In addition, a new model-independent method to determine the amplitude of contributing isobars from the data, rather than using fixed parameterizations, was developed. It was first applied to 
scalar isobars with $J_{\text {iso }}^{P C}=0^{++}$coupling to two pions in an $S$ wave, which play an important role in many decay channels. Strong coupling and isobar phase motions close to $180^{\circ}$, corresponding to the $f_{0}(980)$ and $f_{0}(1500)$, are observed for the $\pi(1800)$ and the $\pi_{2}(1880)$ mother states. The $a_{1}(1420)$ is also seen with this method coupling solely to $f_{0}(980) \pi$, confirming the result with fixed scalar isobar parameterizations. A strong dependence of the shape of the scalar isobar amplitude on the mass of the mother state as well as on $t^{\prime}$ is observed. The new method allows us to reduce the model-dependence of the PWA, albeit at a drastic increase in the number of fit parameters.

Using Primakoff reactions mediated by quasireal photons, the radiative widths of the $a_{2}$ (1320) and, for the first time, the $\pi_{2}(1670)$ were determined to be $\Gamma_{0}\left(a_{2}(1320) \rightarrow \pi \gamma\right)=(358 \pm 6 \pm 42) \mathrm{keV}$ and $\Gamma_{0}\left(\pi_{2}(1670) \rightarrow \pi \gamma\right)=(181 \pm 11 \pm 27) \mathrm{keV} \cdot\left(0.56 / B R_{f_{2} \pi}\right)$, respectively.

\section{References}

[1] J. Beringer et al., Phys. Rev. D 86, 010001 (2012).

[2] R. L. Jaffe, Phys. Rev. D 15, 267 (1977).

[3] J. D. Weinstein and N. Isgur, Phys. Rev. D 27, 588 (1983).

[4] P. Minkowski and W. Ochs, Eur.Phys.J. C9, 283 (1999).

[5] W. Ochs, J. Phys. G 40, 043001 (2013).

[6] E. Klempt and A. Zaitsev, Phys. Rept. 454, 1 (2007).

[7] V. Crede and C. A. Meyer, Prog.Part.Nucl.Phys. 63, 74 (2009).

[8] B. Ketzer, PoS QNP2012, 025 (2012).

[9] S. Aoki et al., Phys.Rev. D79, 034503 (2009).

[10] S. Dürr et al., Science 322, 1224 (2008).

[11] J. J. Dudek et al., Phys. Rev. D 82, 034508 (2010).

[12] J. J. Dudek, Phys. Rev. D 84, 074023 (2011).

[13] P. Abbon et al., Nucl. Instr. Meth. A 577, 455 (2007).

[14] S. U. Chung and T. L. Trueman, Phys. Rev. D 11, 633 (1975).

[15] K. Au, D. Morgan, and M. Pennington, Phys.Rev. D35, 1633 (1987).

[16] J. Basdevant and E. L. Berger, Phys. Rev. D 16, 657 (1977).

[17] V. V. Molchanov et al., Phys. Lett. B 521, 171 (2001).

[18] J. L. Rosner, Phys. Rev. D 23, 1127 (1981).

[19] I. Aznauryan and K. Oganesyan, Sov. J. Nucl. Phys. 47, 1097 (1988).

[20] T. Maeda, K. Yamada, M. Oda, and S. Ishida, arXiv:1310.7507 [hep-ph] (2013). 